\title{
Ex vivo lung perfusion in lung transplantation
}

\author{
Daisuke Nakajima ${ }^{1} \cdot$ Hiroshi Date $^{1}$ \\ Received: 12 November 2020 / Accepted: 17 February 2021 / Published online: 8 March 2021 \\ (c) The Japanese Association for Thoracic Surgery 2021
}

\begin{abstract}
Lung transplantation is an established life-saving intervention for patients with end-stage lung diseases. The success of lung transplantation mainly depends on the quality and function of the implanted donor lungs, which are frequently subject to brain-death-induced lung injuries and intensive care unit (ICU)-related complications before transplantation. Recent innovations, particularly the development of ex vivo lung perfusion (EVLP), in which donor lungs are ventilated and perfused under normothermic conditions outside the body, have allowed clinicians to more accurately assess the donor lung function prior to transplantation. Therefore, EVLP has been successfully translated into clinical practice with the expansion of the donor lung pool, leading to favorable post-transplant outcomes in a growing number of transplant centers worldwide. The EVLP system and techniques, following the Toronto protocol, have recently been applied for the assessment of extended criteria brain-death donors in clinical lung transplantation in Japan. The advancement of EVLP from organ assessment to organ treatment will be the next challenging stage not only to expand donor lung pool, but also to improve graft survival and long-term outcomes after transplantation.
\end{abstract}

Keywords Ex vivo lung perfusion · Organ preservation · Lung transplantation · Extended criteria donor

\section{Introduction}

Lung transplantation is a life-saving procedure for patients with end-stage lung diseases. Donor organ shortage has been exacerbated by the susceptibility of donor lungs to intensivecare unit (ICU)-related complications and injuries acquired during brain death, which have resulted in further obstacles for a clinical use [1]. Thus worldwide donor lung utilization rates were estimated to be only $20 \%$, since many lungs were ultimately turned down for transplantation due to multiple injuries or an uncertain lung function $[1,2]$.

A number of events occurring in donor lungs prior to donor lung recovery, such as brain death, trauma, mechanical ventilation, infection, and aspiration, can exacerbate the ischemia-reperfusion-induced lung injury following lung transplantation [1]. Severe ischemia-reperfusion injury is recognized as a major cause of primary graft dysfunction (PGD), which is associated with early post-transplant

Daisuke Nakajima

daink@kuhp.kyoto-u.ac.jp

1 Department of Thoracic Surgery, Kyoto University Graduate School of Medicine, 54 Shogoin-kawahara-cho, Sakyo-ku, Kyoto 606-8507, Japan morbidity and mortality and an increase in the risk of acute rejection and chronic lung allograft dysfunction (CLAD) [3].

Ex vivo lung perfusion (EVLP) was developed over the past decade to address these issues. EVLP supplies donor lungs with oxygen and nutrients under normothermic conditions $\left(37{ }^{\circ} \mathrm{C}\right)$, which can help lungs sustain active aerobic cellular metabolism ex vivo, keeping them functional for more than $12 \mathrm{~h}[4,5]$. This achievement has positioned EVLP as a platform for evaluating the lung function, treating lung injury, and facilitating lung repair prior to transplantation [6-12].

This article will focus on the clinical application of enhanced EVLP techniques and protocols and the short- and long-term outcomes of lung transplantation with EVLP performed in worldwide transplant centers. The EVLP system was recently introduced in the clinical practice of lung transplantation in Japan to assess the lung functions of extended criteria brain-death donors.

\section{Clinical application of three major EVLP methods}

Three major EVLP systems and protocols are currently being applied in various clinical trials (Table 1): the Toronto protocol using off-the-shelf extracorporeal membrane 
Table 1 Comparison between the clinically used EVLP protocols

\begin{tabular}{llll}
\hline Valuables & Toronto & Lund Vivoline LS1 & OCS \\
\hline $\begin{array}{l}\text { EVLP time } \\
\text { Perfusion }\end{array}$ & $4-6 \mathrm{~h}$ & $2 \mathrm{~h}$ & Transport time \\
Perfusate & STEEN Solution & STEEN Solution + RBC & OCS solu- \\
& Acellular & $($ Hct $14 \%)$ & tion + RBC \\
& & & (Hct 15-25\%) \\
Flow characteristic & Centrifugal pump & Roller pump & Pulsatile pump \\
Target flow & $40 \%$ CO & $100 \%$ CO & 2-2.5 L/min \\
LA pressure $(\mathrm{mmHg})$ & Atrium closed, 3-5 & Atrium open, 0 & Atrium open, 0 \\
Ventilation & ICU ventilator & ICU ventilator & Bellows pump \\
Temperature at start $\left({ }^{\circ} \mathrm{C}\right)$ & 32 & 32 & 32 \\
Tidal volume $(\mathrm{mL} / \mathrm{kg})$ & 7 & $5-7$ & 6 \\
Respiratory rate $(\mathrm{bpm})$ & 7 & 20 & 10 \\
FiO & 0.21 & 0.50 & 0.21 \\
PEEP $\left(\mathrm{cmH}_{2} \mathrm{O}\right)$ & 5 & 5 & 5 \\
\hline
\end{tabular}

OCS Organ Care System, RBC red blood cells, Hct hematocrit, $C O$ cardiac output, $L A$ left atrium, bpm breaths per minute, $P E E P$ positive end expiratory pressure oxygenation (ECMO)-type components and a lung dome and lung cannulas (XVIVO Perfusion AB, Goteborg, Sweden) and acellular perfusate with STEEN Solution (XVIVO Perfusion AB, Goteborg, Sweden), a low potassium extracellular solution including dextran 40 and human albumin, the Lund protocol using the Vivoline LS1 system (Vivoline Medical AB, Lund, Sweden), and the portable Organ Care System (OCS) (Transmedics, Andover, MA, USA) housing a compact mechanical ventilator, pulsatile pump, heat exchanger, and series of monitors.

The Lund EVLP method was clinically used for the first time to evaluate the functions of lungs donated after circulatory death (DCD) in 2000 by Steen [13]. The same group adapted this technique to recondition nine marginal donor lungs primary deemed to be unsuitable for clinical use and reported that six lungs achieved an improved performance, reaching a transplantable quality during short-period EVLP (median EVLP time: $1 \mathrm{~h} 29$ min), which contributed to a significant increase over the previous transplant activity in this group [14].

The first successful clinical trial in Toronto was reported in the New England Journal of Medicine in 2011, demonstrating no significant difference in the early post-transplant outcomes between 20 EVLP-treated lungs and 116 non-EVLP standard lungs: the incidence of PGD grade 2 or 3 at $72 \mathrm{~h}$ was $15 \%$ in the EVLP lungs vs. $30 \%$ in the non-EVLP standard lungs, and the 30-day mortality was $10 \%$ in the EVLP lungs vs. $5 \%$ in the non-EVLP standard lungs [15]. A more recent study has focused on the long-term outcomes of 230 EVLP-treated allografts (24.6\%) of 936 lung transplants performed in the Toronto Lung Transplant Program since 2008, showing that there was no significant difference in the CLAD-free survival (EVLP lungs: 70,56 , and 53\% vs. non-EVLP standard lungs: 72,56 , and $36 \%$ at 3,5 , and 9 years after transplantation, respectively) or allograft survival (EVLP lungs: 73,62 , and $50 \%$ vs. non-EVLP standard lungs: 72,58 , and $44 \%$ at 3,5 , and 9 years after transplantation, respectively) [16].

The portable OCS enables surgeons to quickly initiate perfusion and ventilation of donor lungs at the time point of organ recovery and evaluate the donor lung function during transportation to the designated transplant center. The two international clinical trials using the OCS Lung device have finished, and the results were recently reported. The INSPIRE trial was the first prospective, randomized controlled study for standard bilateral lung transplantation, performed at 21 academic lung transplant centers in the USA, Europe (Belgium, France, Germany, Italy, Spain, and the UK), Australia, and Canada [17]. This study focused on the feasibility and safety of the preservation method using the OCS device for 141 donor lungs meeting the current standard criteria, compared with 165 donor lungs preserved by standard cold storage. Although the incidence of PGD grade 2 or 3 at $72 \mathrm{~h}$ after transplantation was similar between the groups $(11.3 \%$ in the OCS vs. $8.5 \%$ in the standard cold storage), the 30-day mortality was significantly worse in the OCS group (4.2\%) than in the standard cold storage group $(0 \%)$. The EXPAND trial was a prospective single-arm study, performed at eight transplant institutions in the USA, Germany, and Belgium. This multicenter international study showed the safety and efficacy of portable EVLP preservation for 79 transplanted lungs from extended criteria or DCD donors, demonstrating favorable results, with the incidence of PGD 2 or 3 being $16 \%$ at $72 \mathrm{~h}$ post transplantation and the 30-day mortality being $1 \%$ [18]. 


\section{Clinical EVLP experience in Japan}

The first transplant case following the EVLP assessment of the extended criteria donor lungs was reported by the Okayama group in 2015 [19]. EVLP was safely performed and enabled a lobe-specific assessment, which helped identify the left lower lobe as severely injured by aspiration pneumonitis. However, in this case, the donor lungs were ventilated, perfused, and assessed only for a short duration of $89 \mathrm{~min}$.

Since 2018, we have started to re-introduce the clinical practice of an EVLP assessment for extended criteria braindeath donor lungs in lung transplantation in Japan, and we recently experienced two successful transplant cases that required an EVLP assessment of lungs that were initially unacceptable for transplantation.

\section{Entry criteria for EVLP}

EVLP has generally been adopted to expand the donor lung pool through the assessment and reconditioning of extended criteria brain-death donor lungs with worsening or poor oxygenation $\left(\mathrm{PaO}_{2} / \mathrm{FiO}_{2}<300 \mathrm{mmHg}\right)$ due to massive atelectasis or pulmonary edema, poor lung inflation during a direct intraoperative examination at the donor site, pulmonary emboli, or focal consolidation due to infection, aspiration, or lung contusion. However, donor lungs with significant consolidation due to infection, trauma, or gross aspiration deemed unsuitable for transplantation were excluded from the EVLP assessment in most clinical studies [15, 18, 20-22].

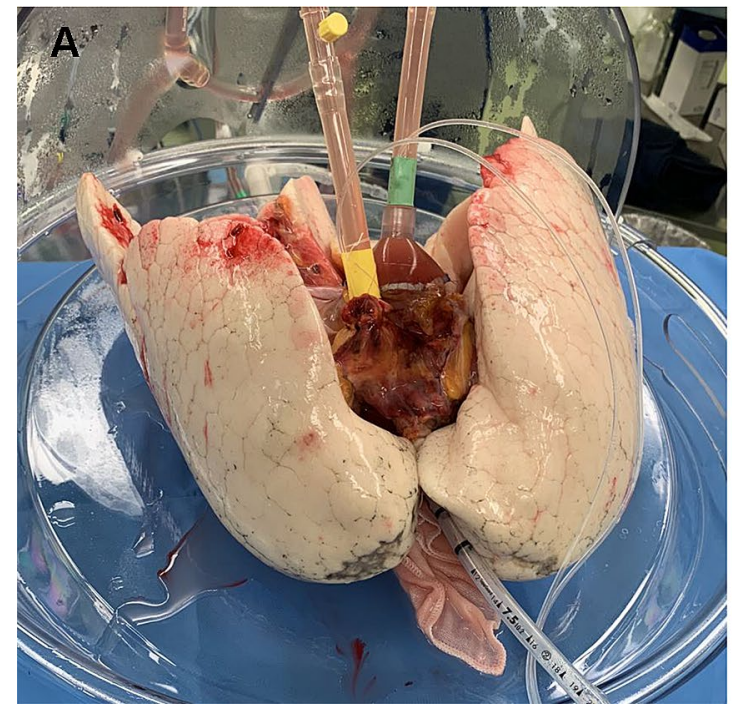

Fig. 1 A green, cone-shaped cannula is attached to the left atrial cuff with a running 4-0 Prolene suture. A yellow straight cannula is inserted into the pulmonary artery and secured with a tie suture. A

\section{The Toronto EVLP procedure}

The Toronto EVLP method was the most widely adopted. On a back table, the pulmonary artery and left atrium were cannulated directly, and the trachea or main bronchus was intubated (Fig. 1), while the EVLP circuit was concurrently primed using STEEN Solution $(1500 \mathrm{ml})$ (XVIVO Perfusion $\mathrm{AB}$, Goteborg, Sweden) with methylprednisolone (500 mg), heparin (10,000 IU), and cefazolin (1 g). After the lung was connected to the EVLP circuit and ventilator (Fig. 2), the perfusate flow was initiated at $10 \%$ of the full flow rate $(40 \%$ of estimated cardiac output) at room temperature. The temperature of the perfusate was gradually increased to $37^{\circ} \mathrm{C}$. When the temperature of the perfusate reached $32{ }^{\circ} \mathrm{C}$, ventilation was started with a $\mathrm{FiO}_{2}$ of 0.21 , tidal volume of $7 \mathrm{ml} /$ $\mathrm{kg}$, frequency of 7 breaths/min, and positive end expiratory pressure (PEEP) of $5 \mathrm{cmH}_{2} \mathrm{O}$, and the deoxygenation of the perfusate was started with a gas mixture of nitrogen (86\%), carbon dioxide (8\%), and oxygen (6\%) at the same time. The perfusate flow rate was gradually increased every $10 \mathrm{~min}$, and the full flow rate was achieved at $50 \mathrm{~min}$ after the start of the perfusion.

\section{Lung assessments during EVLP}

The lung function assessment was initiated at $1 \mathrm{~h}$ after the start of EVLP as a baseline [5, 15]. The physiological lung function, including the pulmonary oxygenation, pulmonary vascular resistance, airway pressures, and pulmonary compliance, should be evaluated every hour for at least $3 \mathrm{~h}$ before a final decision can be made. Pulmonary edema and

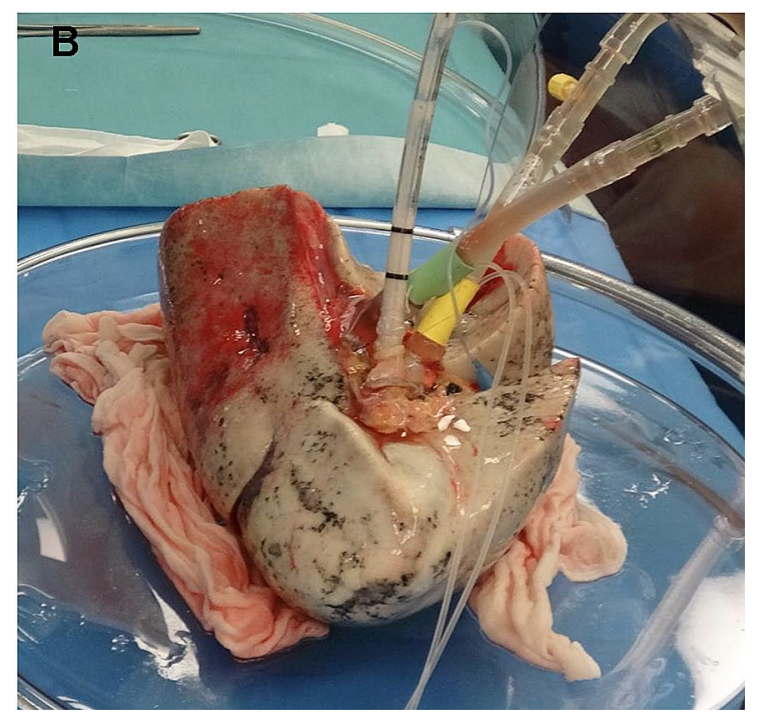

standard endotracheal tube is inserted into the trachea or main-stem bronchus and secured with a tie suture. a Bilateral lung ex vivo lung perfusion (EVLP); b Single-lung EVLP (right lung in this case) 
Fig. 2 The basic components of the EVLP system. In the closed system, the perfusate is circulated by a centrifugal pump (a), passing through a gas exchange membrane (b) and a leukocyte filter (c), and entering the lung. The gas exchange membrane is connected to a heat exchanger (d) and a gas tank (E) that has a deoxygenating gas mixture of nitrogen (86\%), carbon dioxide $(8 \%)$, and oxygen $(6 \%)$. The perfusate flows out of the lung and returns to a hard-shell reservoir (f). The minimal perfusate that might leak from the lung is salvaged from a chamber to the reservoir by a roller pump (g). The lung is ventilated with a ventilator (h)
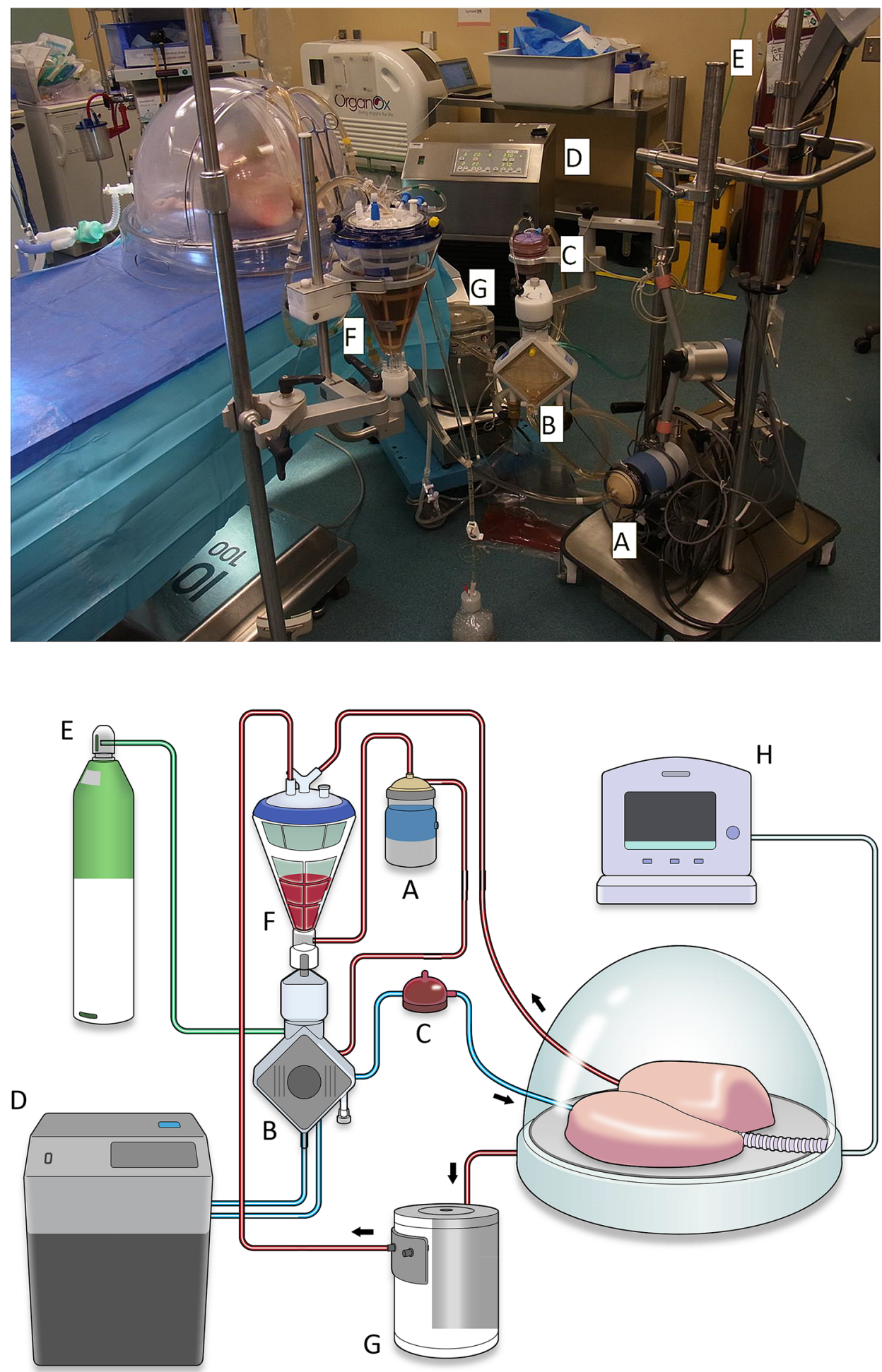

consolidation were also assessed by the bronchoscopy, chest $\mathrm{X}$-ray, and direct palpation of the lungs, performed at the 1 and $3 \mathrm{~h}$ assessments.

When the physiological lung function was improved or stable with no deterioration of $\geq 15-20 \%$ of the baseline value, the delta $\mathrm{pO}_{2}\left(\mathrm{pO}_{2}\right.$ post-lung $-\mathrm{pO}_{2}$ pre-lung $)$ was $\geq 300-400 \mathrm{mmHg}$, pulmonary edema was reduced, and infiltrates or consolidation was still localized without deterioration at the final assessment, the lungs were finally considered suitable for transplantation [15, 18, 20-22]. 


\section{EVLP could safely extend the graft preservation time}

Donor lungs initially deemed unsuitable for transplantation were procured in a standard fashion, transported in static cold storage to the transplant institution, and then connected to the EVLP circuit and ventilator for the assessment.

The Toronto group recently reported that a graft preservation time (including the EVLP time) of over $12 \mathrm{~h}$ (mean CIT1: $4.77 \mathrm{~h}$; mean EVLP time: $5.0 \mathrm{~h}$; mean CIT2: $5.0 \mathrm{~h}$ ) did not negatively affect the early post-transplant outcomes or survival [23]. In that study, the authors successfully transplanted lungs with a total preservation time of up to $21 \mathrm{~h}$, first, CIT of up to $9.5 \mathrm{~h}$, and second, CIT of up to $11 \mathrm{~h}$, without detriment to the overall post-transplant outcomes. This extended preservation time could decrease the geographical limitations for donor hospitals and allow for donor lung recovery over longer distances in Japan [24].

However, according to the results from a recent multicenter study on the pre- and post-EVLP period of CIT, an extended second CIT after EVLP over 287 min was significantly associated with an increase in high-grade PGD at $72 \mathrm{~h}$ after transplantation and 1-year mortality [25]. We also need to keep in mind the disruption of the antegrade bronchial artery circulation during the prolonged organ preservation time [26-28]. Further clinical studies will be needed to determine the maximum safe total preservation time and CIT before and after EVLP.

\section{EVLP as platform for treatment and repair of injuries in donor lungs}

Brain-dead donor lung is subjected to multiple different injuries, caused by brain death, trauma, mechanical ventilation, infection, and aspiration, etc. Each lung needs to be diagnosed and subsequently treated individually in a personalized approach to significantly increase the donor lung pool. The advancement of EVLP from organ assessment to organ repair will be the next challenging step not only to expand donor lung pool, but also to improve graft survival and long-term outcomes after transplantation. The application of enhanced EVLP techniques combined with targeted repairs and molecular therapeutic strategies, including gene and cell-based therapy, will result in improved rehabilitation of injured donor lungs and provide a framework for the application of a personalized medicine approach in lung transplantation $[9,12,29]$. There are several benefits of treating injured donor lungs outside the body: (1) EVLP enables us to perform invasive and time-consuming procedures, which might be difficult in vivo in multi-organ donors with severely deteriorated lung function, (2) EVLP enables us to specifically treat and repair lungs in isolation without collateral toxicity of the treatment to other organs, and (3) EVLP enables us to confirm the effect of treatment and ultimately to transplant an organ with a known and predictable good function. Preclinical studies have started to target and treat different forms of donor lung injury, including infection, aspiration, and pulmonary edema with favorable results [6, $8,11]$. Among them, the clot lysis treatment during EVLP has already been clinically applied for donor lungs injured by pulmonary emboli. There have been three case reports where a fibrinolytic agent (urokinase or alteplase) was administered in the EVLP perfusate to treat donor pulmonary emboli, and the treated lungs were successfully transplanted without any fibrinolytic complications, such as bleeding [30-32].

The potential of EVLP extends beyond lung transplantation and will extend into several areas of medicine, including thoracic oncology. Isolated lung perfusion technique, where the lung is isolated in vivo, can deliver high-dose locoregional chemotherapy with minimal systemic exposure and side effects of antineoplastic drugs. Some clinical trials demonstrated the feasibility and safety of isolated lung perfusion for the treatment of pulmonary metastases [33, 34]. Extended EVLP techniques could make isolated lung perfusion less toxic and more efficient in the high-dose locoregional chemotherapy [35].

\section{Conclusion}

Regardless of different systems and protocols, EVLP has significantly influenced an extended criteria donor lung assessment with the expansion of the donor lung pool and favorable post-transplant outcomes in worldwide transplant centers.

Further development of the EVLP technology is expected to preserve lungs for extended periods, which would provide a larger time window to allow for delivering appropriately targeted therapies and facilitate organ regeneration of injured donor lungs.

\section{Compliance with ethical standards}

Conflicts of interest The authors have no conflicts of interest to disclose.

\section{References}

1. Meers C, Van Raemdonck D, Verleden GM, Coosemans W, Decaluwe H, De Leyn P, et al. The number of lung transplants can be safely doubled using extended criteria donors; a single-center review. Transpl Int. 2010;23:628-35.

2. Israni AK, Zaun DA, Rosendale JD, Snyder JJ, Kasiske BL. OPTN/SRTR 2013 annual data report: deceased organ donation. Am J Transplant. 2015;15(Suppl 2):1-13.

3. de Perrot M, Liu M, Waddell TK, Keshavjee S. Ischemiareperfusion-induced lung injury. Am J Respir Crit Care Med. 2003; 167:490-511. 
4. Cypel M, Rubacha M, Yeung J, Hirayama S, Torbicki K, Madonik $\mathrm{M}$, et al. Normothermic Ex Vivo perfusion prevents lung injury compared to extended cold preservation for transplantation. Am J Transplant. 2009;9:2262-9.

5. Cypel M, Yeung JC, Hirayama S, Rubacha M, Fischer S, Anraku $\mathrm{M}$, et al. Technique for prolonged normothermic ex vivo lung perfusion. J Heart Lung Transplant. 2008;27:1319-25.

6. Nakajima D, Cypel M, Bonato R, Machuca TN, Iskender I, Hashimoto $\mathrm{K}$, et al. ex vivo perfusion treatment of infection in human donor lungs. Am J Transplant. 2016;16:1229-37.

7. Nakajima D, Chen F, Yamada T, Sakamoto J, Ohsumi A, Bando T, et al. Reconditioning of lungs donated after circulatory death with normothermic ex vivo lung perfusion. J Hear Lung Transplant. 2012;31:187-93.

8. Nakajima D, Liu M, Ohsumi A, Kalaf R, Iskender I, Hsin M, et $a l$. Lung lavage and surfactant replacement during ex vivo lung perfusion for treatment of gastric acid aspiration-induced donor lung injury. J Hear Lung Transplant. 2017;36:577-85.

9. Nakajima D, Watanabe Y, Ohsumi A, Pipkin M, Chen M, Mordant $\mathrm{P}$, et al. Mesenchymal stromal cell therapy during ex vivo lung perfusion ameliorates ischemia-reperfusion injury in lung transplantation. J Hear Lung Transplant. 2019;38:1214-23.

10. Lin H, Chen M, Tian F, Tikkanen J, Ding L, Andrew Cheung HY, et al. $\alpha 1$-Anti-trypsin improves function of porcine donor lungs during ex-vivo lung perfusion. J Hear Lung Transplant. 2018;37:656-66.

11. Hijiya K, Chen-Yoshikawa TF, Kondo T, Motoyama H, Ohsumi A, Nakajima $\mathrm{D}$, et al. Bronchodilator inhalation during ex vivo lung perfusion improves posttransplant graft function after warm ischemia. Ann Thorac Surg. 2017;103:447-53.

12. Mordant P, Nakajima D, Kalaf R, Iskender I, Maahs L, Behrens $\mathrm{P}$, et al. Mesenchymal stem cell treatment is associated with decreased perfusate concentration of interleukin- 8 during ex vivo perfusion of donor lungs after 18-hour preservation. J Hear Lung Transplant. 2016;35:1245-54.

13. Steen S, Sjöberg T, Pierre L, Liao Q, Eriksson L, Algotsson L. Transplantation of lungs from a non-heart-beating donor. Lancet. 2001;357:825-9.

14. Ingemansson R, Eyjolfsson A, Mared L, Pierre L, Algotsson L, Ekmehag B, et al. Clinical transplantation of initially rejected donor lungs after reconditioning ex vivo. Ann Thorac Surg. 2009;87:255-60.

15. Cypel M, Yeung JC, Liu M, Anraku M, Chen F, Karolak W, et al. Normothermic ex vivo lung perfusion in clinical lung transplantation. N Engl J Med. 2011;364:1431-40.

16. Divithotawela C, Cypel M, Martinu T, Singer LG, Binnie M, Chow C-W, et al. Long-term Outcomes of Lung Transplant With Ex Vivo Lung Perfusion. JAMA Surg. 2019;154:1143-50.

17. Warnecke G, Van Raemdonck D, Smith MA, Massard G, Kukreja $\mathrm{J}$, Rea F, et al. Normothermic ex-vivo preservation with the portable Organ Care System Lung device for bilateral lung transplantation (INSPIRE): a randomised, open-label, non-inferiority, phase 3 study. Lancet Respir Med. 2018;6:357-67.

18. Loor G, Warnecke G, Villavicencio MA, Smith MA, Kukreja J, Ardehali A, et al. Portable normothermic ex-vivo lung perfusion, ventilation, and functional assessment with the Organ Care System on donor lung use for transplantation from extended-criteria donors (EXPAND): a single-arm, pivotal trial. Lancet Respir Med. 2019;7:975-84.

19. Miyoshi K, Oto T, Konishi Y, Hirano Y, Okada M, Iga N, et al. Use of extended-criteria lungs on a lobe-by-lobe basis through ex vivo lung perfusion assessment. Ann Thorac Surg. 2015;99:1819-21.

20. Aigner C, Slama A, Hötzenecker K, Scheed A, Urbanek B, Schmid W, et al. Clinical ex vivo lung perfusion-pushing the limits. Am J Transplant. 2012;12:1839-47.
21. Sage E, Mussot S, Trebbia G, Puyo P, Stern M, Dartevelle P, et al. Lung transplantation from initially rejected donors after ex vivo lung reconditioning: the French experience. Eur J Cardiothorac Surg. 2014;46:794-9.

22. Zhang ZL, van Suylen V, van Zanden JE, Van De Wauwer C, Verschuuren EAM, van der Bij W, et al. First experience with ex vivo lung perfusion for initially discarded donor lungs in the Netherlands: a single-centre study. Eur J Cardiothorac Surg. 2019;55:920-6.

23. Yeung JC, Krueger T, Yasufuku K, de Perrot M, Pierre AF, Waddell TK, et al. Outcomes after transplantation of lungs preserved for more than $12 \mathrm{~h}$ : a retrospective study. Lancet Respir Med. 2017;5:119-24.

24. Yeung JC, Cypel M, Keshavjee S. Ex-vivo lung perfusion: the model for the organ reconditioning hub. Curr Opin Organ Transplant. 2017;22:287-9.

25. Leiva-Juárez MM, Urso A, Arango Tomás E, Lederer DJ, Sanchez $\mathrm{P}$, Griffith B, et al. Extended post-ex vivo lung perfusion cold preservation predicts primary graft dysfunction and mortality: results from a multicentric study. J Heart Lung Transplant. 2020;39:954-61.

26. Tane S, Noda K, Shigemura N. Ex vivo lung perfusion: a key tool for translational science in the lungs. Chest. 2017;151:1220-8.

27. Tanaka Y, Noda K, Isse K, Tobita K, Maniwa Y, Bhama JK, et al. A novel dual ex vivo lung perfusion technique improves immediate outcomes in an experimental model of lung transplantation. Am J Transplant. 2015;15:1219-30.

28. Shigemura N, Tane S, Noda K. The bronchial arterial circulation in lung transplantation: bedside to bench to bedside, and beyond. Transplantation. 2018;102:1240-9.

29. Cypel M, Liu M, Rubacha M, Yeung JC, Hirayama S, Anraku $\mathrm{M}$, et al. Functional repair of human donor lungs by IL-10 gene therapy. Sci Transl Med. 2009;1:4ra9.

30. Inci I, Yamada Y, Hillinger S, Jungraithmayr W, Trinkwitz M, Weder W. Successful lung transplantation after donor lung reconditioning with urokinase in ex vivo lung perfusion system. Ann Thorac Surg. 2014;98:1837-8.

31. Luc JGY, Bozso SJ, Freed DH, Nagendran J. Successful repair of donation after circulatory death lungs with large pulmonary embolus using the lung organ care system for ex vivo thrombolysis and subsequent clinical transplantation. Transplantation. 2015;99:e1-2.

32. Machuca TN, Hsin MK, Ott HC, Chen M, Hwang DM, Cypel M, et al. Injury-specific ex vivo treatment of the donor lung: pulmonary thrombolysis followed by successful lung transplantation. Am J Respir Crit Care Med. 2013;188:878-80.

33. Den Hengst WA, Hendriks JMH, Balduyck B, Rodrigus I, Vermorken JB, Lardon F, et al. Phase II multicenter clinical trial of pulmonary metastasectomy and isolated lung perfusion with melphalan in patients with resectable lung metastases. J Thorac Oncol. 2014;9:1547-53.

34. Beckers PAJ, Versteegh MIM, Van Brakel TJ, Braun J, Van Putte B, Maat APWM, et al. Multicenter phase II clinical trial of isolated lung perfusion in patients with lung metastases. Ann Thorac Surg. 2019;108:167-74.

35. Dos Santos PR, Iskender I, MacHuca T, Hwang D, Deperrot M, Liu M, et al. Modified in vivo lung perfusion allows for prolonged perfusion without acute lung injury. J Thorac Cardiovasc Surg. 2014; 147:774-81.

Publisher's Note Springer Nature remains neutral with regard to jurisdictional claims in published maps and institutional affiliations. 